\title{
Editorial
}

\section{Multiphase Flow and Transfer Phenomenon}

\author{
Wei Wang, ${ }^{1}$ Panagiota Angeli, ${ }^{2}$ Yueshe Wang, ${ }^{3}$ and Bin $\mathrm{Hu}^{2}$ \\ ${ }^{1}$ Beijing Key Laboratory of Urban Oil \& Gas Distribution Technology, China University of Petroleum, Beijing, China \\ ${ }^{2}$ Department of Chemical Engineering, University College London, Torrington Place, London WC1E 7JE, UK \\ ${ }^{3}$ State Key Laboratory of Multiphase Flow in Power Engineering, Xian Jiaotong University, Xian 710049, China
}

Correspondence should be addressed to Wei Wang; w.wang@cup.edu.cn

Received 23 May 2017; Accepted 23 May 2017; Published 9 July 2017

Copyright (c) 2017 Wei Wang et al. This is an open access article distributed under the Creative Commons Attribution License, which permits unrestricted use, distribution, and reproduction in any medium, provided the original work is properly cited.

The main goal of this special issue was to gather the experimental, theoretical, or numerical research work emphasizing the contributions to the investigation of multiphase flow and heat transfer process. Multiphase flow is widespread in various fields, including power, chemical, petroleum, food, biomedical, metallurgical, nuclear, and hydraulic engineering. Specifically, in oil field, multiphase flow induces many problems ranging from reservoir to pipeline network. Studies on phase distribution and heat or mass transfer are fundamental to give insight into the complicated principle and the application prospect. The papers selected for this topical issue concentrate on numerical work that illustrates the practical problem and optimizes the production process. As such, they provide timely and valuable research results in petroleum field that we are pleased to share with the readers. We would like to thank all the authors for submitting their nice papers and all the referees for their excellent feedback.

This special issue includes five papers, where two papers cover the coalbed methane production, with one related to route optimization and the other concerning production simulation and deliverability forecast. One paper represents the structure optimization of liquid-gas separators by numerical simulation. In addition, one paper simulates the influence of fluid parameters on the vibration of pipeline. Finally, one paper presents both the experimental and simulated observation on the transition criterion from the stratified flow to other flow patterns in gas-hydrate slurry flow.

In the paper entitled "Route Optimization of Pipeline in Gas-Liquid Two-Phase Flow Based on Genetic Algorithm," J. Zhou et al. establish an automatic route optimization model solved by the combination of Generic Algorithm and steadystate Generic Algorithm and discuss the influence of the parameter setting on the results. This method is also applied to determining the pipeline route in coalbed methane field in Shanxi Province, China, and proved to be feasible.

In the paper "Numerical Simulation on Structure Optimization of Liquid-Gas Cylindrical Cyclone Separator," P. Chang et al. represent a three-dimensional turbulence model to describe the multiphase flow field in the cylindrical cyclone separators which is widely used in industrial process and also investigate the dependence of separation efficiency on the separator length, the gas phase outlet diameter, and the inlet shape. Therefore, the optimal geometrical structure of the separator achieving a high efficiency of $86.15 \%$ is given by the author.

In the paper entitled "Study on Influence of Fluid Parameters on Axial Coupled Vibration of Pipeline Conveying Multiphase Flow," M. Chen et al. analyze the effect of the fluid parameters, including void fraction, density ratio, and elastic modulus ratio between solid phase and liquid phase, on the dynamic vibration of the pipe system conveying multiphase flow by numerical models when the valve at the end of pipe is rapidly closed.

In the paper "Coalbed Methane Production System Simulation and Deliverability Forecasting: Coupled Surface Network/Wellbore/Reservoir Calculation," J. Zhou et al. predicts 10 years' water and gas production and formation pressure of a Coalbed methane field with coupled surface/wellbore/reservoir calculation architecture. This paper describes the wellbore pressure drop simulation model, 
surface pipeline network simulation model, and reservoir calculation model of CBM.

In the paper entitled "Investigation on the Transition Criterion of Smooth Stratified Flow to Other Flow Patterns for Gas-Hydrate Slurry Flow," B. Shi et al. develop a stability criterion for gas-hydrate slurry stratified flow with a model based on one-dimensional two-phase flow model and perturbation method after the consideration of some unstable factors including shear stress, gravity, surface tension, and the mass transfer change at the formation of hydrate, and also the criterion is validated by a series of gas-hydrate slurry flow experiments in a high pressure horizontal flow loop.

Wei Wang Panagiota Angeli Yueshe Wang $\mathrm{Bin} \mathrm{Hu}$ 


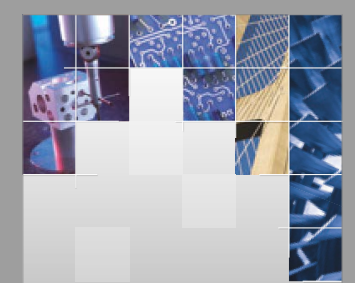

\section{Enfincering}
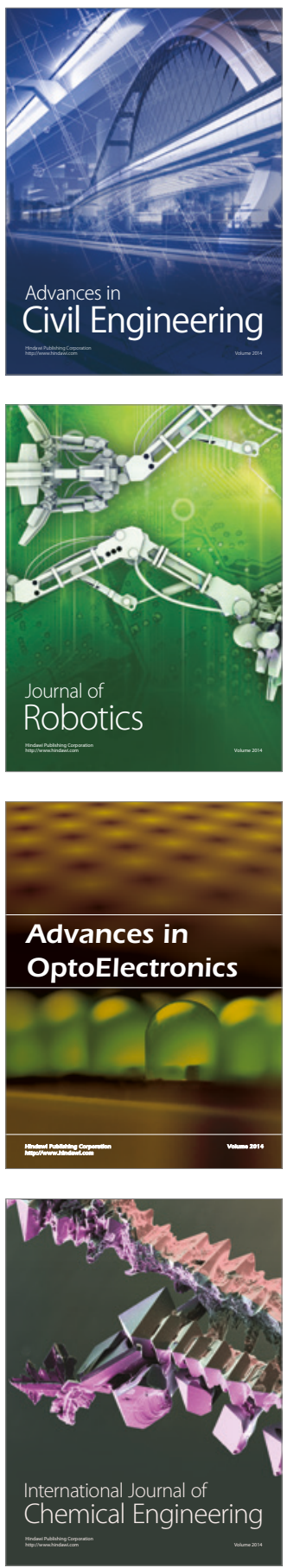

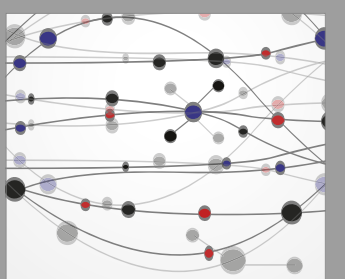

The Scientific World Journal

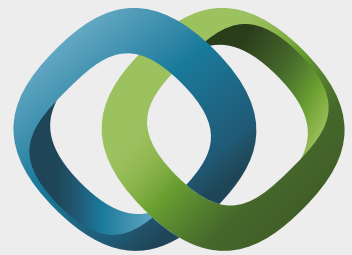

\section{Hindawi}

Submit your manuscripts at

https://www.hindawi.com
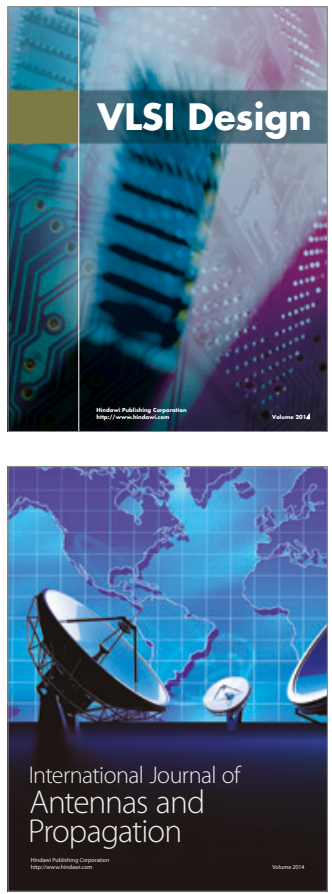

\section{Rotating}

Machinery
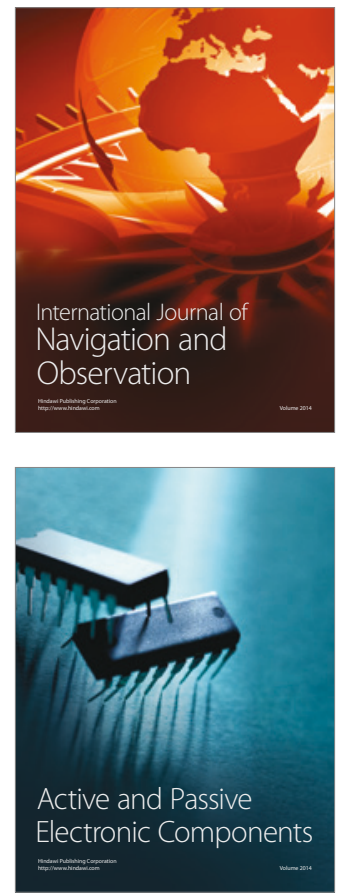
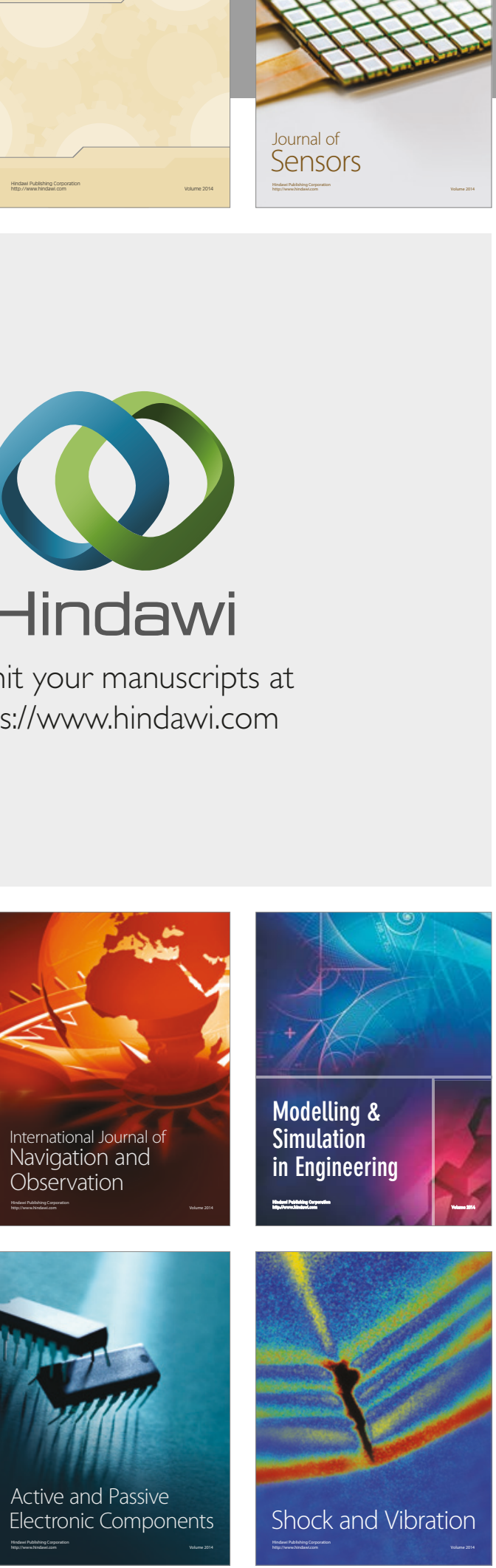
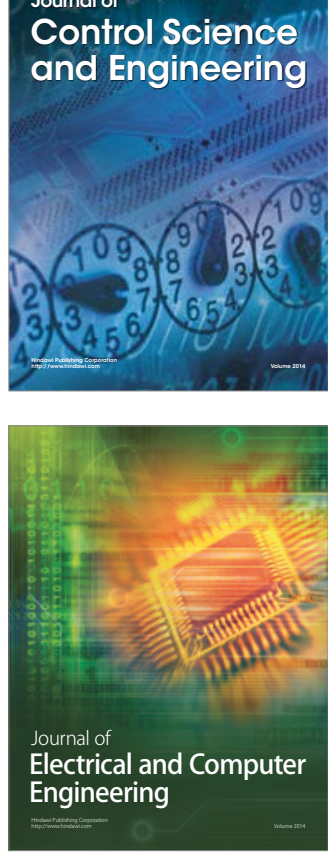

Distributed

Journal of

Control Science

and Engineering
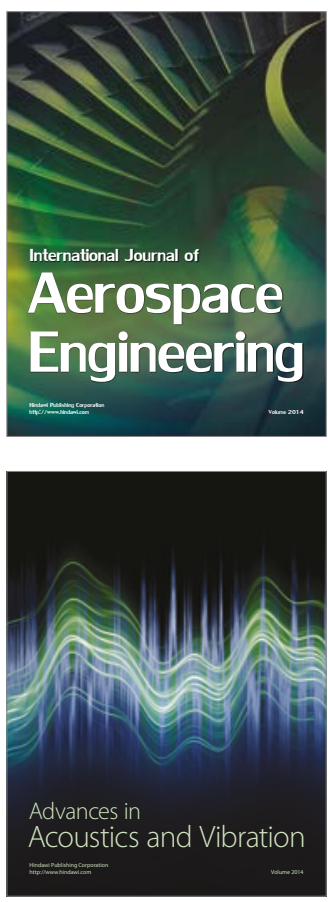

Sensor Networks 